\title{
Four Years' Experience With a Miniaturized Extracorporeal Circulation System and Its Influence on Clinical Outcome
}

\author{
*Christoph Wiesenack, †Andreas Liebold, \$Alois Philipp, *Markus Ritzka, \\ *Joachim Koppenberg, $\$$ Dietrich E. Birnbaum, and §Cornelius Keyl \\ *Department of Anesthesia, University Hospital Regensburg, Regensburg; †Department of Cardiac Surgery, University \\ Hospital Rostock, Rostock; $\$$ Department of Cardiothoracic Surgery, University Hospital Regensburg, Regensburg; and \\ $\S$ Department of Anesthesia, Heart Center Bad Krozingen, Bad Krozingen, Germany
}

\begin{abstract}
It has been suggested that the morbidity associated with cardiopulmonary bypass can be attributed in part to the blood-material and blood-air interactions in the extracorporeal circulation (ECC). A recently introduced minimized ECC-system (MECC System) should be able to reduce these negative effects associated with ECC. A retrospective analysis was performed comprising 485 patients who were operated on for elective coronary artery bypass grafting (CABG) using the MECC System with intermittent antegrade warm blood cardioplegia (group 1) from January 2000 to February 2004. A control group consisted of 485 patients (group 2) undergoing elective CABG in the same period using a conventional ECC and cold crystalloid
\end{abstract}

cardioplegia. There were no significant differences between the two groups in terms of the duration of intubation following surgery, the length of intensive care unitstay and the total hospital stay. Although the 30-day mortality was similar between the two groups, the incidence of postoperative complications and the perioperative use of blood products were significantly higher in the control group compared to the MECC group. The MECC System may serve as an alternative and less invasive approach to conventional ECC. Key Words: Extracorporeal circulation-Minimized extracorporeal circulation-Cardiac surgery-Postoperative complications-Anesthetic management.
Coronary artery bypass grafting (CABG) with extracorporeal circulation (ECC) is widely considered as a safe and effective method for coronary revascularization. Because of the research and development made in cardiopulmonary bypass (CPB), CABG can, at present, be performed with a mortality as low as $3 \%(1,2)$ and thus CABG with ECC represents the gold standard to which any new technique has to be compared. Nevertheless, only about $65 \%$ of conventional CABG procedures reported no complications, and the morbidity of CABG is largely attributed to the use of CPB (3). A systemic inflammatory response syndrome (SIRS) is still associated with $\mathrm{CPB}$, which can result in bleeding, arrhythmias, thromboembolism, endothelial dysfunction, neurological disorders, or organ dysfunction $(3,4)$. In order to minimize these deleterious effects of $\mathrm{CPB}$, the use

Received March 2004; revised July 2004.

Address correspondence and reprint requests to Dr. Christoph Wiesenack, University Hospital, Department of Anesthesia, Franz-Josef-Strauss Allee 11, 93052 Regensburg, Germany. E-mail: christoph.wiesenack@klinik.uni-regensburg.de of off-pump coronary artery bypass grafting $(\mathrm{OPCAB})$ has gained renewed interest as a potentially safer technique to maintain the functional integrity of major organ systems. Postulated benefits of OPCAB procedures include a reduction in morbidity, lower cost resulting from a shorter hospital stay and convalescence period, an equivalent quality of the bypass grafts and increased patient comfort, compared to traditional CABG with ECC $(5,6)$. However, despite a high level of experience, OPCAB has been reported to cause incomplete revacularization and a higher rate of early re-operation (7). Furthermore, hemodynamic collapse may occur during OPCAB because of ischemic or mechanical complications and result in a total hospital mortality of about $2 \%(8)$. Suitable patient selection is essential for successful OPCAB procedures. Nevertheless, $\mathrm{CPB}$ will still be needed for many procedures in cardiac surgery. In Germany, currently about $94 \%$ of all CABGs are performed using ECC, thus a reduction of the invasive aspects of $\mathrm{CPB}$ is still of real interest (9). 
Recently a modified ECC-system was introduced and first described by Philipp and colleagues, which initially was conceived to assist circulation in beating heart procedures (10). Later on, its application was extended for CABG with cardioplegic cardiac arrest using intermittent antegrade warm blood cardioplegia.

The minimal extracorporeal circulation-system (MECC System, Jostra AG, Hirrlingen, Germany) is a closed, fully heparin-coated (Bioline Coating, Jostra AG) and preconnected ECC-system, consisting of a diffusion membrane oxygenator (Quadrox D, Jostra AG) and a centrifugal pump (RotaFlow, Jostra AG). The reduction of the tubing length and the removal of a venous reservoir and cardiotomy suction prevent any air-blood contact in combination with a high grade of biocompatibility. This new system should be able to reduce the negative effects associated with conventional ECC, such as systemic inflammatory reaction, hemolysis, hemodilution, disturbances of blood clotting disorders, and postoperative complications.

This retrospective study was performed to analyze 4 years' experience with the MECC System regarding the clinical outcome and the incidence of postoperative complications.

\section{MATERIALS AND METHODS}

After obtaining approval from the local Ethics Committee, a retrospective analysis was performed at a single university hospital using a prospectively collected surgical database comprising 1909 cardiac surgical patients undergoing elective CABG between January 2000 and February 2004. Among these patients, 485 elective CABG patients were identified who were operated on using the MECC System with intermittent warm antegrade blood cardioplegia (group 1, MECC). During the same period, 485 control cases were randomly chosen from the same database. These patients underwent elective CABG using a conventional ECC and cold crystalloid cardioplegic solution (group 2, ECC). The same surgeons, anesthesiologists and perfusionists were involved in both groups of patients. Patients with a relevant aortic valve insufficiency or a body mass index $(\mathrm{BMI})>40 \mathrm{~kg} / \mathrm{m}^{2}$ were excluded from the MECC procedure.

Anesthesia was induced in all patients with intravenous Fentanyl (Janssen-Cilag GmbH, Neuss, Germany) $5 \mu \mathrm{g} / \mathrm{kg}$, followed by Etomidate (B. Braun Melsungen AG, Melsungen, Germany) until loss of consciousness and Pancuronium (Curamed Pharma $\mathrm{GmbH}$, Karlsruhe, Germany), $100 \mu \mathrm{g} / \mathrm{kg}$, and main- tained either with an infusion of Propofol (Astra Zeneca GmbH, Wedel, Germany) of 3-5 mg/kg/h, or $0.4-0.8 \%$ endexspiratory Isoflurane (Abbott $\mathrm{GmbH}$, Wiesbaden, Germany), supplemented with bolus doses of Fentanyl up to $10 \mu \mathrm{g} / \mathrm{kg}$ and Pancuronium, $30 \mu \mathrm{g} / \mathrm{kg}$. In both groups Aprotinin (Bayer Vital $\mathrm{GmbH}$, Leverkusen, Germany) was administered prior to institution of CPB in a dose of at least $2 \times 10^{6}$ kallikrein inhibitory units when platelet active medication was given less than 6 days before elective surgery.

The MECC System is a fully heparin-coated and preconnected closed ECC system consisting of a diffusion membrane oxygenator and a centrifugal pump at a nonpulsatile flow rate of up to $2.6 \mathrm{~L} / \mathrm{min} / \mathrm{m}^{2}$, a two-stage venous cannula (40-32 Fr), an aortic cannula $(7 \mathrm{~mm})$, and $3 / 8$ inches PVC tubing $(180 \mathrm{~cm})$ as shown in Fig. 1. When required, online blood gas and saturation monitoring (CDI 500, Terumo, Tokyo, Japan) could be incorporated in the venous or arterial line of the system. Integrated in the drive unit of the centrifugal pump were a flow meter and a bubble sensor. With the help of a heat exchanger as part of the oxygenator, mild hypothermia during bypass to a rectal temperature of $33-34^{\circ} \mathrm{C}$ and rewarming of the patient were easily achieved. The reduction of the tube length and the removal of a venous reservoir, an arterial filter, and cardiotomy suction prevented any air-blood contact and allowed a reduced priming volume of about $480 \mathrm{~mL}$ ( $280 \mathrm{~mL}$ of Ringer's solu-

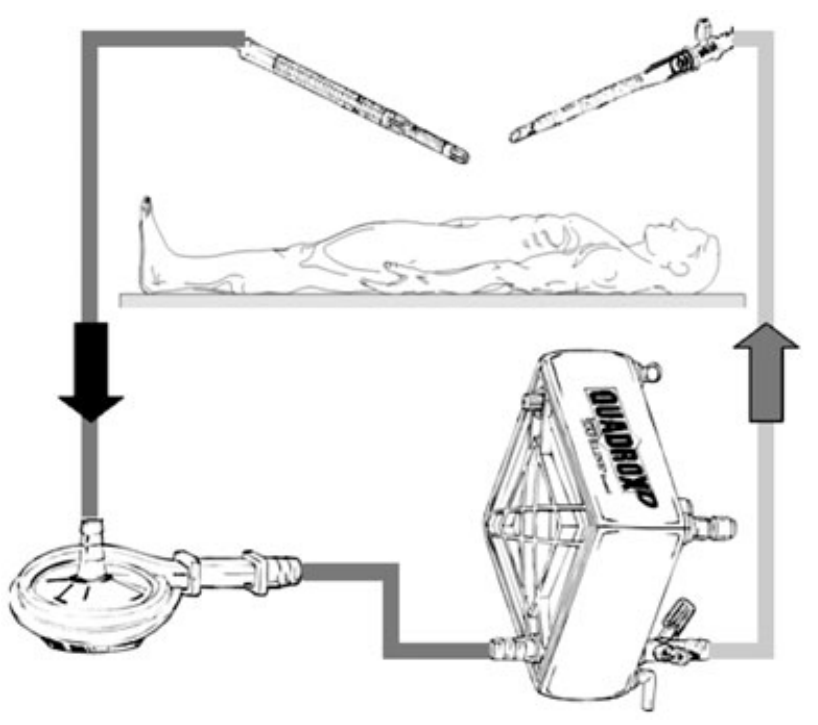

FIG. 1. Composition of the minimal extracorporeal circulation system (MECC): closed-loop system without venous reservoir, consisting of a diffusion membrane oxygenator and a centrifugal pump. 
tion and $200 \mathrm{~mL}$ of mannitol 20\%). A shuntline was integrated in the circuit to provide arterial blood sampling and volume or drug administration during bypass. Before initiating the MECC System, anticoagulation was attained by administration of $150 \mathrm{IU} /$ $\mathrm{kg}$ heparin to achieve an activated clotting time (ACT) of 250-300 s. A cell-saving device was used for blood salvaging from the surgical field and retransfusion of the centrifuged and washed blood at the end of surgery.

CABG in group 1 was performed using a modified Calafiore cardioplegia technique (11). Cardioplegic solution (mixture of $30 \mathrm{~mL} \mathrm{KCl} 14.9 \mathrm{Vol} \%$ and $6 \mathrm{~mL}$ $\mathrm{MgSO}_{4} 50 \mathrm{Vol} \%$ ) was delivered intermittently by an antegrade route via an aortic root cannula with vent line and pressure tubing (DLP, Medtronic, Minneapolis, MN, U.S.A.) with the cardioplegia line exiting the cardioplegia port of the oxygenator. After initiating MECC and aortic cross clamping, ventricular fibrillation was induced.

In the control group, a standard CPB technique was used for all patients. Before aortic cannulation, patients were anticoagulated with heparin (375 IU/ $\mathrm{kg}$ ) to an ACT of $>400 \mathrm{~s}$. A two-stage cannula was used for drainage of venous blood from the right atrium. The bypass circuit consisted of a roller pump (Stöckert Instruments, Munich, Germany) at a nonpulsatile pump flow rate of $2.6 \mathrm{~L} / \mathrm{min} / \mathrm{m}^{2} \pm 10 \%$ and one of the following three microporous membrane oxygenators, providing nearly equivalent gas transfer characteristics: Capiox RX25 (Terumo, Tokyo, Japan), Hilite7000 (Medos, Stolberg, Germany), Quadrox-Safeline (Jostra AG). The circuit was primed with a balanced crystalloid/colloid solution (1000 mL of Ringer's solution, $200 \mathrm{~mL}$ of mannitol $20 \%$, and $300 \mathrm{~mL}$ of $6 \%$ HES 200/0.5) and consisted of a hard shell venous reservoir. Alpha stat blood gas management was used. After aortic cross clamping and single dose administration of $1500 \pm 400 \mathrm{~mL}$ cold cardioplegic solution (Custodiol HTKBretschneider, Dr. Franz Köhler Chemie AlsbachHähnlein, Germany), a stable level of perfusion pressure with mild hypothermia (rectal temperature 33$34^{\circ} \mathrm{C}$ ) was obtained.

\section{Morbidity parameters analyzed}

Blood samples were taken for maximum values of lactate concentration during bypass and minimum values of hemoglobin, measured subsequent to cardioplegia application.

Postoperative myocardial infarction was assessed by serial electrocardiographic changes and a typical rise and fall in the serum creative kinase MB (CK$\mathrm{MB})$ activity. An increase in plasma creatinine of
$>20 \%$ from preoperative value within 3 days after surgery defined postoperative renal insufficiency. Patients required dialysis in case of oligoanuria, hyperkalemia, and metabolic acidosis. Stroke was defined as a new neurologic deficit confirmed by computer tomography or magnetic resonance imaging.

\section{Statistical analysis}

Statistical analysis was performed using the SPSS 10.0 software (SPSS, Chicago, IL, U.S.A.). Normal distribution was assessed by the Lilliefors modification of the Kolmogorov-Smirnov test. Values of continuous data are presented as mean $\pm \mathrm{SD}$ or as median (range) when appropriate. Categorical variables are displayed as frequency distributions $(n)$ and simple percentages (\%). Univariate analysis of variance (ANOVA) with a posthoc Scheffeé test was performed for specific differences between the groups for normally distributed continuous variables and with the Kruskal-Wallis test, followed by the Mann-Whitney $U$-test for not normally distributed continuous variables. Univariate comparison between the groups for categorical variables was made using the $\chi^{2}$ and the Fisher's exact test when appropriate. Within-group comparisons of continuous data were performed using a general linear model procedure (repeated-measures analysis of variance). Statistical significance was considered when $P<0.05$.

\section{RESULTS}

Both groups were similar with respect to demographic data and preoperative risk factors (Table 1).

Differences between the MECC-group and the control group regarding the intraoperative data are presented in Table 2. In particular, the number of grafts $(3.3 \pm 0.8$ vs. $3.5 \pm 0.9)$, the duration of CPB ( $79 \pm 24 \mathrm{~min}$ vs. $86 \pm 24 \mathrm{~min}$ ), the time of aortic cross clamping (48 $\pm 15 \mathrm{~min}$ vs. $51 \pm 16 \mathrm{~min})$, and the minimal measured temperature during bypass $\left(33.6 \pm 1.0^{\circ} \mathrm{C}\right.$ vs. $\left.33.0 \pm 1.2^{\circ} \mathrm{C}\right)$ were similar in both groups. Cardioplegia volume $(22 \pm 7 \mathrm{~mL}$ vs. $1519 \pm$ $449 \mathrm{~mL}, P<0.05)$ and ACT measured at onset of CPB $(362 \pm 96 \mathrm{~s}$ vs. $561 \pm 163 \mathrm{~s}, P<0.05)$ were significantly lower in group 1 compared to group 2. Mean arterial pressure (MAP) and mean pump flow rate during bypass differed significantly between the groups at all timepoints as shown in Fig. 2. The frequency of vasoactive drug administration was significantly lower in MECC-patients compared to ECC-patients (318 [65\%] vs. 429 [88\%], $P<0.05$ ).

Maximum values of lactate concentration during bypass were significantly higher in the control group 
TABLE 1. Demographic data and preoperative risk factors

\begin{tabular}{lccc}
\hline & $\begin{array}{c}\text { Group 1 } \\
\text { MECC } \\
(n=485)\end{array}$ & $\begin{array}{c}\text { Group 2 } \\
\text { ECC } \\
(n=485)\end{array}$ & $P<0.05$ \\
Variable & $66 \pm 8.6$ & $65 \pm 8.6$ & n.s. \\
\hline $\begin{array}{l}\text { Demographic data } \\
\text { Age (year) }\end{array}$ & $351 / 134$ & $385 / 100$ & n.s. \\
Sex (male/female) & 27.6 & 20.6 & \\
$(\%$ female) & $28 \pm 4.0$ & $28 \pm 3.9$ & n.s. \\
BMI (kg/m $)$ & $63 \pm 15$ & $62 \pm 16$ & n.s. \\
EF $(\%)$ & & & \\
Preoperative risk factors & $145(30 \%)$ & $136(28 \%)$ & n.s. \\
Diabetes & $310(64 \%)$ & $334(69 \%)$ & n.s. \\
Hypertension & $126(26 \%)$ & $145(30 \%)$ & n.s. \\
Smoking & $291(60 \%)$ & $305(63 \%)$ & n.s. \\
Hyperlipidemia & $247(51 \%)$ & $242(50 \%)$ & n.s. \\
Obesity & $39(8 \%)$ & $34(7 \%)$ & n.s. \\
History of stroke & $73(15 \%)$ & $82(17 \%)$ & n.s. \\
PVD & $82(17 \%)$ & $87(18 \%)$ & n.s. \\
COPD & $64(13 \%)$ & $68(14 \%)$ & n.s. \\
Renal disease & &
\end{tabular}

Data are presented as mean \pm SD or as frequency distributions $(n)$ and simple percentages (\%). BMI, body mass index; EF, left ventricular ejection fraction; PVD, peripheral vascular disease; COPD, chronic obstructive pulmonary disease.; n.s., not significant.

$(14.1 \pm 6.2 \mathrm{mg} / \mathrm{dL})$ compared to the MECC group $(11.1 \pm 4.7 \mathrm{mg} / \mathrm{dL}, P<0.05)$. Minimum values of hemoglobin as an indicator of hemodilution differed significantly between group 1 and group 2 $(10.2 \pm 1.4 \mathrm{~g} / \mathrm{dL}$ vs. $7.6 \pm 2.6 \mathrm{~g} / \mathrm{dL}, P<0.05)$. There were no significant differences between the two groups regarding the duration of intubation follow-

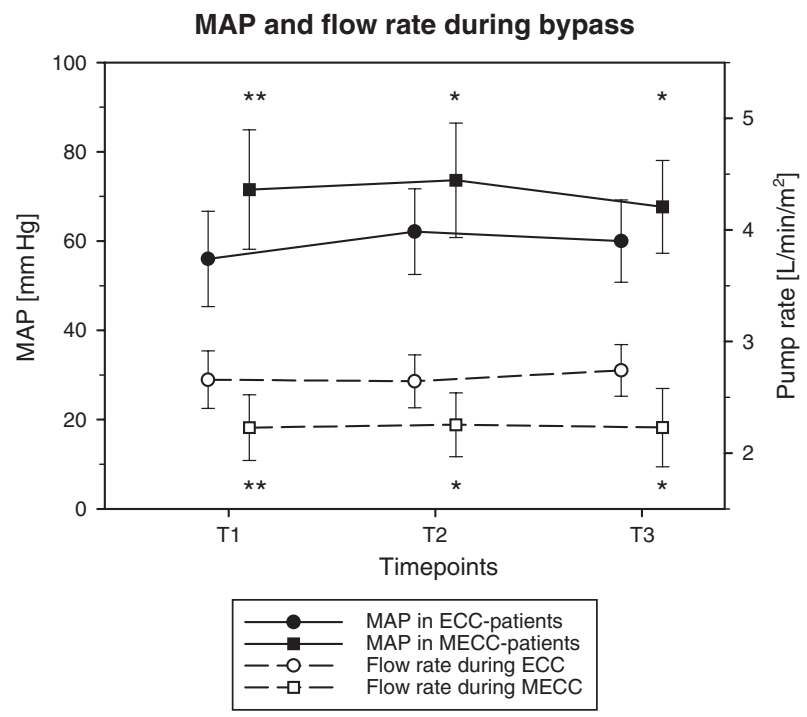

FIG. 2. Mean arterial pressure (MAP) and pump flow rate during bypass in both groups at timepoints: $\mathrm{T} 1$ = following cardioplegia administration, $\mathrm{T} 2=$ in the middle of bypass time at $34^{\circ} \mathrm{C}$, T3 $=$ end of aortic cross clamping time. ${ }^{*} P<0.05$ for MECC vs. ECC. ing surgery, the length of intensive care unit (ICU) stay and the total hospital stay (Table 2).

While postoperative blood loss was comparable between the two groups, the number of patients receiving transfusion of packed red blood cells (PRBCs) during surgery (150 [31\%] vs. 42 [8.6\%]) and during the complete perioperative course (383 [79\%] vs. 190 [39\%]) was significantly larger in the control group compared to the MECC group $(P<0.05)$.

Clinical outcomes and the incidence of postoperative complications are detailed in Table 2. Whereas the 30-day mortality was similar between both groups, the incidence of postoperative complications was significantly higher in the control group compared to the MECC group $(P<0.05)$.

TABLE 2. Perioperative data

\begin{tabular}{|c|c|c|c|}
\hline Variable & $\begin{array}{c}\text { Group 1 } \\
\text { MECC } \\
(n=485)\end{array}$ & $\begin{array}{c}\text { Group } 2 \\
\text { ECC } \\
(n=485)\end{array}$ & $P$ value \\
\hline \multicolumn{4}{|l|}{ Intraoperative data } \\
\hline Grafts $(n)$ & $3.3 \pm 0.8$ & $3.5 \pm 0.9$ & n.s. \\
\hline CPB-time (min) & $79 \pm 24$ & $86 \pm 24$ & n.s. \\
\hline $\mathrm{X}$-clamp time $(\mathrm{min})$ & $48 \pm 15$ & $51 \pm 16$ & n.s. \\
\hline Temperature $\min \left({ }^{\circ} \mathrm{C}\right)$ & $33.6 \pm 1.0$ & $33.0 \pm 1.2$ & n.s. \\
\hline Cardioplegia $(\mathrm{mL})$ & $22 \pm 7$ & $1519 \pm 449$ & * \\
\hline $\operatorname{ACT}(\mathrm{s})$ & $362 \pm 96$ & $561 \pm 163$ & * \\
\hline Use of Phenylephrine $(n)$ & $318(65 \%)$ & $429(88 \%)$ & $*$ \\
\hline Lactate preoperative & $9.1 \pm 4.3$ & $9.0 \pm 3.9$ & n.s. \\
\hline$(\mathrm{mg} / \mathrm{dL}) \max$ & $11.1 \pm 4.7$ & $14.1 \pm 6.2$ & * \\
\hline $\mathrm{Hb}$ preoperative & $12.6 \pm 1.5$ & $12.8 \pm 2.1$ & n.s. \\
\hline$(\mathrm{g} / \mathrm{dL}) \min$ & $10.2 \pm 1.5$ & $7.6 \pm 2.6$ & * \\
\hline \multicolumn{4}{|l|}{ Postoperative data } \\
\hline Ventilation (h) & 11 [2-216] & 12 [2-242] & n.s. \\
\hline ICU stay (day) & $1[0-32]$ & $1[0-38]$ & n.s. \\
\hline Hospital stay (day) & $12[1-65]$ & $13[2-38]$ & n.s. \\
\hline \multicolumn{4}{|l|}{ Hemostatic data } \\
\hline \multicolumn{4}{|l|}{ PRBC } \\
\hline OR & $42(8.6 \%)$ & $150(31 \%)$ & * \\
\hline Total & $190(39 \%)$ & $383(79 \%)$ & $*$ \\
\hline FFP & $86(17 \%)$ & $80(16 \%)$ & n.s. \\
\hline Chest tube drainage $(\mathrm{mL})$ & $765 \pm 606$ & $817 \pm 619$ & n.s. \\
\hline \multicolumn{4}{|c|}{ Mortality and postoperative complications } \\
\hline 30-day mortality & $8(1.6 \%)$ & $12(2.5 \%)$ & n.s. \\
\hline Myocardial infarction & $9(1.9 \%)$ & $16(3.3 \%)$ & * \\
\hline Low cardiac output & $4(0.8 \%)$ & $21(4.3 \%)$ & $*$ \\
\hline Atrial fibrillation & $58(12 \%)$ & $160(33 \%)$ & * \\
\hline Defibrillation & $19(3.9 \%)$ & $49(10 \%)$ & * \\
\hline Re-thoracotomy & $1(0.2 \%)$ & $15(3.1 \%)$ & * \\
\hline Re-intubation & $9(1.9 \%)$ & $25(5.1 \%)$ & * \\
\hline Pneumonia & $4(0.8 \%)$ & $15(3.1 \%)$ & * \\
\hline Renal insufficiency & $4(0.8 \%)$ & $15(3.1 \%)$ & $*$ \\
\hline Dialysis & $1(0.2 \%)$ & $11(2.2 \%)$ & * \\
\hline Stroke & $5(1.0 \%)$ & $15(3.1 \%)$ & * \\
\hline
\end{tabular}

Data are presented as mean $\pm \mathrm{SD}$, as median (range) or as frequency distributions $(n)$ and simple percentages (\%) when appropriate. ACT, activated clotting time; ICU, intensive care unit; PRBC, packed red blood cells; OR, operating room; FFP, fresh frozen plasma; $\mathrm{Hb}$, hemoglobin; min, minimum value, measured following cardioplegia administration; n.s., not significant.

$* P<0.05$ for MECC vs. ECC. 


\section{DISCUSSION}

The results of our observation demonstrate a comparable postoperative course regarding the length of ICU stay, total hospital stay, and 30-day mortality, together with a significantly lower incidence of postoperative complications in the MECC group compared to the control group, indicating that the MECC System can serve as an alternative and less invasive approach to conventional ECC.

In the present study, maximum values of lactate concentration (normal, $0.0 \mathrm{mg} / \mathrm{dL}$ to $20.0 \mathrm{mg} / \mathrm{dL}$ ) during bypass were significantly higher in the control group compared to the MECC group (Table 2). Though the interpretation of elevated lactate concentrations is limited by several confounding variables, measurement of blood lactate levels is widely used to assess the adequacy of tissue perfusion (12). Based on regional blood flow and lactate exchange measurements, Takala et al. stated that hyperlactatemia after cardiac surgery is a sign of inadequate or marginal tissue perfusion of the hepatosplanchnic region, as well as other tissues (12). The differences between the groups in the present study regarding lactate concentration were slight, but statistically significant (Table 2) and confirmed the impression that the MECC System can be considered to be less detrimental concerning organ failure compared to conventional ECC and to provide a better tissue perfusion, as shown by the incidence of postoperative complications (Table 2). This might be in part the effect of the volume-constant perfusion featured in the closedloop MECC System regularly resulting in an elevated MAP compared to conventional CPB particularly at the onset of circulation as shown in Fig. 2. The increased perfusion pressure and the greater intravascular volume, resulting from the removal of a venous reservoir, together with the consequently decreased need for vasoactive drugs during bypass, should provide a better capillary perfusion of all organs (Table 2).

It has also been suggested that the morbidity associated with CPB can be attributed in part to SIRS caused by blood-material and blood-air interactions in the ECC (13). Biocompatible circuits designed to prevent the early activation of inflammatory cascades have been shown to affect various aspects of blood activation and appear promising. Improvements in the design of the ECC by integrating different components with minimal surface area and diminished blood-air interactions should further minimize the blood activation. Fromes et al. recently demonstrated that the MECC System provides a lower inflammatory reaction when compared to standard CPB (14).

Bioline Coating of the MECC System combines polypeptide and active heparin to simulate a natural endothelium. This coating method should ensure stable bonding of the heparin molecule and the immobilized polypeptide. The effects of heparincoated circuits on the inflammatory response during CPB have been extensively studied and it has been shown that heparin coating could decrease complement activation and cytokine production and attenuates the release of neutrophil granular proteases in CPB $(13,15,16)$. However, other studies showed controversial results, indicating no benefit when using heparin-coated circuits $(17,18)$. Therefore, it is difficult to solely examine the effect of heparin coating on blood activation, because many other factors independent of ECC contribute to blood trauma.

In the MECC group anticoagulation was attained by administration of $150 \mathrm{IU} / \mathrm{kg}$ heparin to achieve an ACT of $250-300 \mathrm{~s}$, corresponding to only $40 \%$ of the usually administered heparin dose. This recommendation stands in contrast to Fromes et al. who described a heparin dose of $300 \mathrm{IU} / \mathrm{kg}$ for the MECC procedure (14). According to the reduced heparin dose in the fully heparin-coated MECC circuit, ACT at onset of $\mathrm{CPB}$ was significantly lower in the MECC group compared to the control group (Table 2). Ovrum et al. measured various coagulation and fibrinolysis parameter in patients undergoing $\mathrm{CABG}$ with full (ACT > 480 s) and reduced (ACT > $250 \mathrm{~s}$ ) systemic heparinization and concluded that CABG can be safely performed with reduced systemic heparinization in combination with fully heparin-coated circuits (19). No anticoagulation complications were detected at any time in the study period; therefore we found it permissible to reduce the systemic heparinization to $150 \mathrm{IU} / \mathrm{kg}$ heparin in the fully heparincoated circuit of the MECC System.

Another source of blood activation is a direct blood-air interface. Schönberger and colleagues noticed more systemic blood activation during and after CPB in patients treated with an open venous reservoir than in those treated with a closed reservoir with the consequence of an increased blood loss (20). The venous reservoir is accused of being the foremost source of activated blood and recirculation of shed blood should therefore be precluded (21). The removal of a venous reservoir and cardiotomy suction in the closed MECC circuit prevents any air-blood contact and contributes to this experience. Furthermore, the significantly reduced pump flow rate of the MECC System as a result of the 
increased MAP during the MECC procedure (Fig. 2), should result in a decreased amount of hemolysis. Although only a statistically not significant tendency toward a higher postoperative blood loss in the ECC group could be observed in the present investigation, the intra- and postoperative use of blood products was significantly higher in the control group compared to the MECC group and confirms these findings.

The reduction of the tube length and the removal of a venous reservoir, arterial filter, and cardiotomy suction prevent not only any air-blood interface but also allow a reduced priming volume of about $480 \mathrm{~mL}$. Minimum values of hemoglobin as an indicator of hemodilution clearly verified this effect, as a significant difference could be observed between the control group and the MECC group (Table 2). Because of the absent venous reservoir, the MECC System is only compatible with small volumes of cardioplegia. The higher cardioplegia volume used in the conventional bypass group in contrast to the small amounts of Calafiore solution in group 2 further pronounced hemodilution.

The use of a centrifugal pump instead of a roller pump in addition to the incorporation of a new diffusion membrane oxygenator instead of a conventional membrane oxygenator should reduce the mechanical trauma to blood cells as well as blood activation (22). Therefore, such a new oxygenator containing a plasma-tight poly(4-methyl-1-pentene) membrane was incorporated in the MECC System to provide optimal biocompatibility. The wall of the new plasma-tight hollow fiber consists of a highly porous support matrix and a thin $(0.05 \mu \mathrm{m})$ tight membrane on the blood side of the matrix. This membrane constitutes a solid barrier between blood and gas, and is therefore also described as a solid or diffusion membrane. The homogenous nonporous membrane and the complete separation of blood and gas phase should provide a better biocompatibility with less blood traumatization. Crossing of micro bubbles caused by a lowered pressure on the blood side compared to the gas side as well as plasma leakage should not occur because of the tightness of the membrane $(23,24)$.

The application of volatile agents into the gas supply line of the MECC System is possible but technically demanding. However, recent studies demonstrated a markedly decreased uptake of Isoflurane into blood via the new poly (4-methyl-1pentene) membrane oxygenators compared to conventional polypropylene membrane oxygenators $(23,24)$. Therefore, Propofol may be preferable for maintenance of anesthesia in MECC patients to ensure a constant level of the applied anesthetic agent. However, the possible loss of ability to use volatile agents which cause preconditioning of the myocardium could be a major potential disadvantage of the system (25).

The MECC System employs kinetically assisted venous drainage, which raises some safety issues, particularly the increased risk of air entrainment. Therefore, a bubble sensor is integrated in the drive unit of the centrifugal pump, which will trigger a visual alarm. Furthermore, the pressure in the venous line is monitored continuously to avoid a negative pressure in the right atrium. Nevertheless, the attentiveness of the perfusionist is essential to stop and deair the circuit via the shuntline in the case of air entrainment to prevent air embolism. Alternatively, incorporation of a soft reservoir bag should provide easy management of air entrainment.

\section{Limitations}

Even if both groups appear comparable and had a relatively equal distribution of all significant preoperative variables, the data are retrospective and this is a limitation of the study. Although we made every attempt to acknowledge all clinically significant variables and to eliminate any selection bias, the analytical technique accounts only for identified variables and it is possible that an effect may exist from an unquantified variable.

\section{CONCLUSIONS}

In the present study we found a comparable postoperative course regarding the length of ICU-stay, total hospital stay and 30-day mortality, together with a significantly lower incidence of postoperative complications in patients undergoing CABG with the help of the MECC System compared to patients undergoing CABG with full circulatory support. Despite the limitations mentioned above, the results of the present study demonstrated that the MECC System may serve as an alternative and less invasive approach to conventional ECC. The results found in our observation should be confirmed in prospective trials, to evaluate above all, whether a specific group of patients with preexisting organ dysfunctions could benefit from the new system.

Acknowledgments: Departmental funding supported this study financially. The authors wish to acknowledge the support for this work provided by the Department of Anesthesia and Department of Cardiothoracic Surgery, University Hospital, Regensburg, Germany. 


\section{REFERENCES}

1. Edwards FH, Clark RE, Schwartz M. Coronary artery bypass grafting: The Society of Thoracic Surgeons National Database experience. Ann Thorac Surg 1994;57:12-9.

2. Fitzgibbon GM, Kafka HP, Leach AJ, Keon WJ, Hooper GD, Burton JR. Coronary bypass graft fate and patients outcome: Angiographic follow-up of 5065 grafts related to survival and reoperation in 1388 patients during 25 years. J Am Coll Cardiol 1996;28:616-26.

3. Borst C, Gründemann PF. Minimally invasive coronary artery bypass grafting. An experimental perspective. Circulation 1999;99:1400-3.

4. Royston D. The inflammatory response and extracorporeal circulation. J Cardiothorac Vasc Anesth 1997;11:341-54.

5. Puskas JD, Williams WH, Duke PG, et al. Off-pump coronary artery bypass grafting provides complete revacularization with reduced myocardial injury, transfusion requirements, and length of stay: A prospective randomized comparison of two hundred unselected patients undergoing off-pump versus conventional coronay artery bypass grafting. J Thorac Cardiovasc Surg 2003;125:797-808.

6. Nathoe HM, van Dijk D, Jansen EW, et al. A comparison of on-pump and off-pump coronary bypass surgery in low-risk patients. N Engl J Med 2003;348:394-402.

7. Arom KV, Flavin TF, Emery RW, Kshettry VR, Janey PA, Petersen RJ. Safety and efficacy of off-pump coronary artery bypass grafting. Ann Thorac Surg 2000;69:704-10.

8. Vassiliades TA Jr, Nielsen JL, Lonquist JL. Hemodynamic collapse during off-pump coronary artery bypass grafting. Ann Thorac Surg 2002;73:1874-9.

9. Kalmar P, Irrgang E. Cardiac surgery in Germany during 2001: A report by the German Society for Thoracic and Cardiovascular Surgery. Thorac Cardiovasc Surg 2002;50:30-5.

10. Philipp A, Foltan M, Thrum A, et al. MECC - a minimal ECC-system for coronary artery bypass procedures. $J$ Extra Corpor Technol 2002;34:A215.

11. Calafiore AM, Teodori G, Mezzetti A, et al. Intermittent antegrade warm blood cardioplegia. Ann Thorac Surg 1995;59:398-402.

12. Takala J, Uusaro A, Parviainen I, Ruokonen E. Lactate metabolism and regional lactate exchange after cardiac surgery. New Horiz 1996;4:483-92.

13. Hsu LC. Biocompatibility in cardiopulmonary bypass. J Cardiothorac Vasc Anesth 1997:11:376-82.

14. Fromes Y, Gaillard D, Ponzio O, et al. Reduction of the inflammatory response following coronary bypass grafting with total minimal extracorporeal circulation. Eur J Cardiothorac Surg 2002;22:527-33.

15. Palatianos GM, Foroulis CN, Vassili MI, et al. A prospective, double-blind study on the efficacy of the bioline surfaceheparinized extracorporeal perfusion circuit. Ann Thorac Surg 2003;761:129-35.

16. Ovrum E, Mollnes TE, Fosse E, et al. Complement and granulocyte activation in two different types of heparinized extracorporeal circuits. J Thorac Cardiovasc Surg 1995;110:162332.

17. Muehrcke DD, McCarthy PM, Kottke-Marchant K, et al. Biocompatibility of heparin-coated extracorporeal bypass circuits: A randomized, masked clinical trial. J Thorac Cardiovasc Surg 1996;112:472-83.

18. Wildevuur CR, Jansen PG, Bezemer PD, et al. Clinical evaluation of Duraflo II heparin treated extracorporeal circulation circuits (2nd version). The European Working Group on heparin coated extracorporeal circulation circuits. Eur J Cardiothorac Surg 1997;114:616-23.

19. Ovrum E, Brosstad F, Am Holen E, Tangen G, Abdelnor M. Effects on coagulation and fibrinolysis with reduced versus full systemic heparinization and heparin-coated cardiopulmonary bypass. Circulation 1995;92:2579-84.

20. Schönberger JP, Everts PA, Hoffmann JJ. Systemic blood activation with open and closed venous reservoir. Ann Thorac Surg 1995;59:1549-55.

21. Brown Mahoney C, Donnelly JE. Impact of closed versus open venous reservoirs on patients outcome in isolated coronary bypass surgery. Perfusion 2000;15:467-72.

22. Moen O, Fosse E, Dregelid E, et al. Centrifugal pump and heparin coating improves cardiopulmonary bypass biocompatibility. Ann Thorac Surg 1996;62:1134-40.

23. Philipp A, Wiesenack C, Behr R, Schmid FX, Birnbaum DE. High risk of intraoperative awareness during cardiopulmonary bypass with isoflurane administration via diffusion membrane oxygenators. Perfusion 2002;17:175-8.

24. Wiesenack C, Wiesner G, Keyl C, et al. In vivo uptake and elimination of isoflurane by different membrane oxygenators during cardiopulmonary bypass. Anesthesiology 2002;97:1338.

25. Julier K, da Silva R, Garcia C, et al. Preconditioning by sevoflurane decreases biochemical markers for myocardial and renal dysfunction in coronary artery bypass graft surgery: A double-blinded, placebo-controlled, multicenter study. Anesthesiology 2003;98:1315-27. 